\title{
Enhancing Integrated Indoor/Outdoor Mobility in a Smart Campus
}

Joaquín Torres-Sospedra, Joan Avariento, David Rambla, Raúl Montoliu, Sven Casteleyn, Mauri Benedito-Bordonau, Michael Gould, and Joaquín Huerta

Institute of New Imaging Technologies, Universitat Jaume I, Castellón, Spain

Corresponding author: Joaquín Torres-Sospedra

Email: jtorres@uji.es

Present address: Institute of New Imaging Technologies, Universitat Jaume I, Avda. Vicente Sos Baynat S/N, E-12071, Castellón, Spain. 


\title{
Enhancing Integrated Indoor/Outdoor Mobility in a Smart Campus
}

\author{
A Smart City relies on six key factors: Smart Governance, Smart People, Smart \\ Economy, Smart Environment, Smart Living and Smart Mobility. This paper \\ focuses on Smart Mobility by improving one of its key components: positioning. \\ We developed and deployed a novel indoor positioning system that is combined \\ with an outdoor positioning system to support seamless indoor and outdoor \\ navigation and wayfinding. The positioning system is implemented as a service \\ in our broader cartography-based smart university platform, called SmartUJI, \\ which centralizes access to a diverse collection of campus information and \\ provides basic and complex services for the Universitat Jaume I (Spain), which \\ serves as surrogate of a small city. Using our indoor positioning system and \\ based on the SmartUJI services, we developed, deployed and evaluated two end- \\ user mobile applications: the SmartUJI APP that allows users to obtain map- \\ based information about the different facilities of the campus, and the SmartUJI \\ $A R$ that allows users to interact with the campus through an augmented reality \\ interface. Students, university staff and visitors who tested the applications \\ reported their usefulness in locating university facilities and generally improving \\ spatial orientation.
}

Keywords: Smart University, Mobility, Indoor Positioning, Map-based Navigation, Augmented Reality

\section{Introduction}

During the last years there has been a shift from primarily rural to primarily urban population, and this trend is projected to continue in the next couple of decades (Chourabi et al. 2012). This generates new challenges in cities which range from transparent government and better use of scarce resources, to energy and waste management and environmental pollution, to mobility and transportation issues (Borja 2007, Marceau 2008, and Washburn et al. 2010). According to Chourabi et al. (2012), modern cities and megacities require a deeper understanding of the interconnected issues, challenges, and actors under the umbrella of the Smart City concept. 
There are many approaches that embrace the idea of Smart City (Cohen 2012, and Greco \& Bencardino 2014). For example, Vienna is significantly reducing energy consumption and emissions without having to forego any aspects of consumption or mobility $^{1}$. The private initiative SmartCommute ${ }^{2}$ is just one way in which the City of Toronto works with businesses to reduce traffic congestion and harmful carbon emissions by encouraging the use of sustainable transportation modes. London puts Londoners at the heart of the city driven by the principles of transparency, collaboration and efficiency ${ }^{3}$. Fujisawa city, in the Tokyo area, is developing a state-of-art sustainable town ${ }^{4}$ based on actual lifestyles that focuses on self-consumption of energy, ensuring safety, providing eco-friendly mobility, improving health, and supporting community life.

These examples illustrate that there is no unique solution or approach to realize the concept of Smart City. Each city is different in size, population and geography, and is built upon a unique socio-economic and cultural substrate. As such, the heterogeneity in needs and requirements makes it difficult to come up with a unique, unambiguous definition of Smart City. Nevertheless, there are some common issues and needs that most cities share. The concept of Smart City thus emerges from these shared needs, which are reflected by Giffinger and colleagues (Giffinger et al. 2007, and Giffinger et al. 2008), who proposed a widely adopted framework to compare the strategies used and the current deployment of Smart Cities (e.g., McCarthy \& Vickers 2008, and Nam \& Pardo 2011, and $\operatorname{IEEE}^{5}$ ). By conducting an extensive analysis of European mediumsized cities, the authors identify the following six key, self-descriptive fields: Smart

\footnotetext{
${ }^{1} \mathrm{http}: / / \mathrm{smartcity}$. wien.at/site/en/

${ }^{2}$ http://www.smartcommute.ca/

${ }^{3} \mathrm{http}: / /$ www.london.gov.uk/priorities/business-economy/vision-and-strategy/smart-london

${ }^{4} \mathrm{http} / / /$ fujisawasst.com/EN/project/

${ }^{5} \mathrm{http}: / /$ smartcities.ieee.org/about.html
} 
Governance, Smart People, Smart Economy, Smart Environment, Smart Living and Smart Mobility. These fields reflect the main areas of interest shared by many cities, and allow for easy comparison.

Similar as for Smart Cities, researchers have attempted to characterize the concept of Smart University as a set of dimensions or key fields. Although cities and universities are distinct environments with different objectives, they often share a similar socio-economic, environmental and geographical context (i.e., a university is usually placed in/nearby a city), and so they share similar services, infrastructures, communication channels, transport networks, and even challenges and needs. As such, Coccoli et al. (2014) emphasize education and knowledge sharing as defining dimensions of a Smart University, but it also covers other key areas such as Communication, Transport, and Governance. Along the same lines, Ng et al. (2010) suggested the term Intelligent Campus articulated along six main axes: iLearning, iGovernance, iGreen, iHealth, iSocial, and iManagement. For Owoc and Marciniak (2013), Smart People, Smart Building, Smart Environment, Smart Governance and Knowledge grid are the five elements of a Smart University.

The brief analysis of the main dimensions of a Smart City and a Smart University suggests that there indeed exists a significant overlap of the priority areas and dimensions that define cities and university campuses. A university campus covers a scenario where thousands of people (students, employees and visitors) go about their daily activities, and can thus function as a surrogate small city or city neighbourhood.

In this paper we focus on the Smart mobility dimension. An essential requirement of any smart application that aims to enhance mobility is knowing the user's actual position, both outdoor and indoor. While outdoor positioning is usually obtained using GPS, precise and accurate indoor positioning systems are currently only 
scarcely available. Therefore, our main contribution presented in this paper to enhance mobility is the deployment of a proprietary Indoor Positioning System (IPS) that computes and monitors the actual indoor position of a user and integrate it with an outdoor positioning system to provide seamless indoor and outdoor positioning and wayfinding. In order to be universally deployable, we considered it essential that our IPS be infrastructure-less, i.e., not require any additional hardware to be installed. This IPS is thus based on existing Wi-Fi signals, and is realized as a service in a broader, open smart university platform called SmartUJI, which is a modular, extensible cartography-based platform to store, access and manage all the data, resources, common services and functionalities required to build a wide range of applications in a smart campus context. Two such applications related to mobility are presented here. The first, the SmartUJI App, allows users to search and obtain information about the different campus facilities using a map-based interface, and facilitates pedestrian navigation and wayfinding. The second application, the $\operatorname{Smart} U J I A R$, offers similar functionality, but leverages an augmented reality video-based interface (rather than a map-based view) to provide the users with a more immersive experience, showing routes and point-ofinterest icons visible through the users' mobile device camera. The SmartUJI platform, including IPS and the mobile apps, was deployed and evaluated in the context of Universitat Jaume I (UJI), which, with around 18.000 people in a centralized campus, is a real-life testing environment for Smart Mobility developments.

\section{Related work}

Coccoli et al. (2014) reviewed Smart University experiments which can be considered successful. Expenses and insurance costs were notably reduced at 5 medical universities and 10 campuses by adopting the risk management system developed by the University of California. Infrastructure and maintenance costs have been reduced in North Carolina 
State University by using cloud computing systems. Furthermore, the first step taken by some universities is to reduce energy consumption and reinvest the savings in improved teaching. Owoc and Marciniak (2013) also mentioned that knowledge management was a crucial piece of a Smart University.

As mentioned in $\mathrm{Ng}$ et al. (2013), not every key factor need be implemented or developed to qualify as a Smart University. One key factor that is not mentioned in descriptions of smart campuses, yet was a key part of our project, is the creation of a software platform which can support multiple mobile applications (apps). Here we focus on mobility apps by introducing an indoor positioning system and smart applications to support pedestrian navigation and wayfinding. Mobility in the previous Smart City and Smart University projects rarely considers indoor pedestrian navigation as we do. Moreover, the Smart Campus initiatives do not normally integrate the different Smart Projects they offer in a common platform to homogenize access to data and services.

Although the inclusion of $A-G P S$ in modern phones has solved the problem of outdoor positioning, performing indoor location is still an interesting research topic since GPS has severe limitations inside buildings. Several technologies are being used to deal with the indoor location problem: Radio-Frequency Identification (Jin et al. 2006), Bluetooth (Feldmann et al. 2006), Wireless Local Area Network, Wi-Fi, (Bahl and Padmanabhan 2000, Gansemer et al. 2010, Machaj et al. 2011, and Marques et al. 2013), ZigBee (Martí et al. 2012), ultrasonic (Ijaz et al. 2013), magnetic (Chung et al. 2011), and even LED light-based (Kuo et al. 2014) among others. Nowadays, Wi-Fi and magnetic field mapping are two of the most promising technologies for indoor location due to Wi-Fi connectivity and the embedded sensors in smartphones. 


\section{Smart Campus Use Case}

The Universitat Jaume I (UJI) was created in 1991 and offers its state-regulated instruction on a modern campus of approximately $176,000 \mathrm{~m}^{2}$. The campus is designed as a single-walled space in order to encourage closer human relationships, and is equipped with up-to-date academic, research, cultural and social facilities. It is a green, sustainable campus that promotes energy saving and the use of renewable energies, with among others recycling points facilitating the recovery of materials and monitoring sensors for energy consumption. It is also easily accessible, with public transportation running through the campus, and designed without any architectural barriers to be fully accessible for disabled people. It effectively functions as a modern small city.

In this context, we identify emphasis on several key areas of smart cities and smart universities, as described in Section 1: smart people, environment and mobility (Giffinger et al. 2007), transport (Coccoli et al. 2014), smart building (Owoc and Marciniak 2013), iGreen and iSocial (Ng et al. 2010). Our research group has been developing tools and applications that reinforce the smartness of the UJI campus, focusing on those concepts different from the main university's objectives related to learning, research, development and innovation:

- Monitor utilities consumption (energy, water, gas) and detect anomalies

- Improve user-environment relations and enhance human satisfaction

- Air quality and weather monitoring

- Promote sustainable mobility

As we came to realize, a key success criterion for any application truly exploiting and connecting different aspects of a city/university context is managing and providing unified access to a variety of heterogeneous and decentralised information. 
Such basic functionality, realized as services, is provided by the SmartUJI platform, where all the public information related to the university has been standardized and made accessible using RESTful and Web Services.

\section{SmartUJI platform}

The SmartUJI platform relies on a three-tier architecture, where data, services and applications are well differentiated (see Figure 1). To build this platform, we mainly used commercial off-the-shelf GIS software (e.g. ArcGIS Server) similar to that used in many cities.

\section{[Figure 1 near here]}

The data layer contains the structure and relationships of all relevant data generated in the university to realize a smart campus. Typically, university activities require and produce a huge quantity of data. However, as is often the case, the available data at UJI is scattered in several databases, and/or stored in different formats. Most academic information is stored in relational databases, but some, such as measurements collected by consumption sensors, rely on non-relational databases. Moreover, some information is provided as independent text and/or binary files (e.g., txt, $d o c, p d f$, or $d x f$ ). To deal with this heterogeneity, the data layer keeps track of the different data, their location and access method(s). The data layer thus supports local data, stored and managed within the SmartUJI platform, and external data, stored and managed externally. For example, databases can be local or external; in both cases an ODBC or JDBC bridge would suffice as access method. One important (local) data source is the ArcGIS server that contains the geospatial content, which is essential to provide a cartographic view on the campus. Other data sources include, but are not limited to data related to energy consumption (external non-relational database), university facility data (external 
relational databases and external AutoCad files), etc.

The service layer contains the services that are available to end-user application builders, and offers specific functionalities related to the smart campus. An important group of services are the information services, which provide unified access to data sources in the data layer. The SmartUJI platform provides unified access, update, visualization, creation, publication and elimination services to all data sources that reside in the data layer. Information services are interfaced by the ArcGIS REST API specification $^{6}$, which enables application developers and services to access and interact with the SmartUJI platform's information services in a single, uniformed manner based on resource-oriented interfaces. Furthermore, changes in internal data models and data management practices only require corresponding changes in the platform, and so are completely transparent to the end-user applications and their developers because information services' interfaces are kept intact. As such, the smart campus platform is a middleware between end-user applications and different data sources, thus acting as data access coordinator. On top of the information services, other services offer specific, specialized functionality to application developers. While being specific, these services are not tied to a particular application but can be part of various types of end-user applications addressing, for example, mobility and environment dimensions. Some services may however exploit platform or device specific features (e.g., the Indoor Positioning System uses the Wi-Fi information of a mobile device), in which case they only work on supported platforms/devices. Examples of specific services include:

Finder: allows searching for a specific university member, subject, space, department, or facility. It consumes other services to access external data sources and the internal geospatial contents to link the results to locations on the map.

\footnotetext{
${ }^{6} \mathrm{http}: / /$ resources.arcgis.com/en/help/arcgis-rest-api/
} 
Route calculation: calculates the optimal route for a set of points. This functionality has been implemented for three dimensions (longitude, latitude and altitude) to reach any space, interior or exterior, on the campus using stairways. This service uses geoprocessing capabilities through the information services.

Speech recognizer: supports the interaction of users with mobile applications, and is thus an example of a specific (device-dependent) service. It is able to distinguish 4279 relevant campus locations which were extracted from the SmartUJI platform. The automatically generated semantic models include only the relevant words for Spanish, Valencian, and English (Granell et al. 2014).

Finally, the application layer corresponds to the end-user applications that consume the platform services and process/expose the data and/or functionality provided by them. An example of end-user applications other than the smart mobile apps described in Section 6 we developed is the SmartUJI web portal client ${ }^{7}$.

Due to its architecture and design choices, the SmartUJI platform is modular, scalable and multi-platform. All data and services can be accessed and invoked through standard interfaces, and from any platform ${ }^{8}$. The Indoor Positioning System is a specific (device-dependent) service in the SmartUJI platform designed to improve mobility on campus (see Section 5), and is exploited by two mobile end-user applications (see Section 6): SmartUJI APP and SmartUJI AR.

\section{Indoor Positioning System for Mobile Devices}

Mobile devices have an interesting feature which is typically not included in desktopbased applications: sensors that allow a precise positioning. Knowing the position of the device, and therefore the position of the user who is holding it, is crucial in context-

\footnotetext{
${ }^{7}$ http://smart.uji.es/

${ }^{8}$ Except those exploiting device or platform specific features, as explained before.
} 
aware applications based on location. Although a browser-IP-based location system is used in web-based applications to locate the user, the accuracy provided by GPS and IPSs is much higher.

Therefore, a novel Wi-Fi-based fingerprint based Indoor Positioning System (IPS) has been integrated as a service into the SmartUJI platform. As the IPS is realized as a service, it is external to the applications, and any improvement on its accuracy is transparent to the mobile application developers and users. The IPS provides an estimation of the user's position (operational phase), based on the current Wi-Fi fingerprint captured through an end-user mobile application running on the user's mobile device. To do so, the IPS compares the current fingerprint to a database of reference fingerprints, obtained during the calibration phase.

\subsection{Calibration: Indoor Mapping}

To generate the indoor database of reference fingerprints, a Wi-Fi mapping procedure was carried out inside the buildings. This mapping consisted of capturing the Wi-Fi signals at a set of previously selected reference points, using a mobile app specifically designed for this purpose. As a result of mapping the buildings of the School of Technology and Experimental Sciences, we obtained and published the UJIIndoorLoc database (Bache and Lichman 2013, and Torres-Sospedra et al. 2014), whose main characteristics are:

- It covers a surface higher than $100,000 \mathrm{~m}^{2}$, including 3 buildings with $4 / 5$ floors.

- Data were collected by more than 20 users using 25 different devices.

- 520 different Wireless Access Points (WAPs) were found.

- The fingerprint reference database contains a total of 21048 fingerprints, of which 1111 were used for testing. 


\subsection{Operation: Full Positioning}

For the operational phase, the indoor location system uses a two-step strategy. First, the system estimates the sector of the campus where the current fingerprint (provided by the user's mobile application upon localization request) is located using a probabilisticbased estimator. To do so, the campus area was divided into a regular grid, where each cell was assigned to a campus sector. In the second step, the IPS uses $k$-Nearest Neighbors, kNN, Cover and Hart (1967), to estimate the current position of the current fingerprint based on the reference database. The system hereby only considers those fingerprints inside the estimated sector, thereby avoiding comparison with all reference fingerprints, thus keeping the computational costs reasonable while also improving accuracy. This IPS was deployed in the SmartUJI platform servers. An example of full location is shown in Figure 3C, where the arrow icon shows the position of the user.

\section{Smart mobile applications to improve mobility}

On a campus, just as in a city, spatial awareness and mobility are often difficult when one is not within the familiar context. On the UJI campus, spaces and facilities are alphanumerically coded to uniquely identify them. However, the correspondence between these codes and the real-world spaces they identify are notoriously difficult to understand, and thus provide little help. Furthermore, the UJI campus contains over 30 singular buildings, each with their own particularities and complex coding system, which makes navigating the campus a non-trivial task.

SmartUJI APP and SmartUJI AR have been developed to deal with the above mentioned problem and improve mobility of the user. We focus on spatial awareness and orientation, support to spatially locate resources (buildings, rooms, employees), and wayfinding. The applications address the following target audiences: 
- new students or visitors who are not familiar with the campus facilities;

- students and staff who do not know or do not remember where some building or office is located, and/or cannot correctly interpret the complex coding system;

- reduced-mobility users who want to know where some special services, such as ramps and special parking spots, are located.

Both applications are freely available at Google Play ${ }^{9,10}$ and the research group's repository. They provide similar functionality: search for and visualize information about university facilities, navigation and wayfinding. Their main distinctive feature is the user interface and user interaction: while SmartUJI APP visualizes information using an intuitive map-based view, the SmartUJI AR uses an augmented reality interface whereby interactive icons are superimposed on campus facilities and observed by the user through the mobile device camera. Moreover, the SmartUJI APP has been designed to allow the integration of other modules, such as calendars, class schedules, or chat rooms, which are not useful in an AR-based application. The two applications are described in detail in the following two subsections.

\subsection{SmartUJI APP}

The SmartUJI APP is an Android application that shows the campus map along with some relevant information on it. The application accesses the geospatial content provided by the SmartUJI platform and provides map visualization capabilities using the ArcGIS Runtime SDK for Android. The information to display is selected by the user with an intuitive menu or by using a layer selector that groups the information in categories like Transportation, Parking, or Facilities, among others.

\footnotetext{
${ }^{9}$ https://play.google.com/store/apps/details?id=es.uji.smartuji.base \&hl=en

${ }^{10} \mathrm{https}$ ://play.google.com/store/apps/details?id=com.metaio.SmartUjiAR\&hl=en
} 
The application also allows the user to search for a staff member, office, classroom, campus facility or building by entering the person's name, or the official building/facility/room's name. The application returns a list of matches, shows all useful information for them, and locates them on the map. For example, searching for a staff member's name (e.g., "Huerta") returns all staff members who have "Huerta" in their name - see Figure 2A. Once the correct result was selected (i.e., "Joaquin Huerta" in our example), the information related to this staff member is visualized - see Figure 2B. The search functionality in this app is realized using the SmartUJI's Finder service, while map visualization is realized using the Map Access service (see section 4).

To further improve mobility within the campus, the application also offers Location Based Services (LBS). The main functionality of the application is the seamless indoor/outdoor map-based navigation that is realized using the Route Calculation service to calculate the best route between two points, and uses the Indoor Positioning System service described in this paper (see Section 5) if the user is indoors, and the Global Navigation Satellite System (e.g., GPS) service if the user is outdoors. Figure 2C shows an example of such navigation, where SmartUJI APP guides the user from the current position to Joaquin Huerta's office. Indoor and outdoor positioning systems are seamlessly integrated, and indoor/outdoor navigation is visually indicated: the solid line indicates the route in the current floor (in our example, the 5th floor), whereas the dotted line refers to parts of the route on other floors and outside the building. As the user changes floors, the lines are updated to indicate the path in the current floor. The arrow indicates the current user's current position and orientation.

[Figure 2 near here]

\subsection{SmartUJI AR}

SmartUJI AR is an Android application allowing the user to visualize the location of the 
nearby points of interest (recycling bins in Figure 3A) and facilities (the Library in Figure 3A) with an Augmented Reality (AR) viewer, as well as to guide them through the campus while the route is displayed in real time (see the indoor route to a research laboratory in Figure 3B). This application has been developed using MetaioSDK, which is an augmented reality API for mobile devices, in combination with relevant services provided by the SmartUJI platform.

[Figure 3 near here]

With this interactive tool, the user is able to visually locate and filter (see the Figure 3C) the location of facilities and points of interest. This is realized by calling SmartUJI services, and visualized on the camera view of the mobile phone using MetaioSDK. Other features included are location-based searches and facilities location, and visualizing additional information about any visualized facility, all realized using the SmartUJI services. Also included is a module that simulates the experience of being on the campus when the user is elsewhere, using pre-captured $360^{\circ}$ images and locations.

Finally, SmartUJI AR allows the user to calculate the route to any university's facility (through the SmartUJI Route Calculation service) from the current location, by jointly using the SmartUJI IPS service for indoor localization and the GPS sensor for outdoor location. As the SmartUJI AR offers similar functionality as SmartUJI APP, they exploit common SmartUJI services, and only the client code is different: where SmartUJI APP uses map visualization services, SmartUJI AR uses MetaioSDK to visualize information on top of the mobile phone's camera view.

\section{Evaluation and user experiences}

\subsection{IPS: Evaluation setup and results}

For testing, we tried to mimic a real-world scenario as closely as possible. The 
SmartUJI Indoor Positioning System was deployed in an infrastructure-less environment, and it only used the already available wireless signals to provide the user's location. No additional hardware was installed, e.g. to increase Wi-Fi coverage. The fingerprint training (calibration) and test sets were obtained by different users using different devices, 4 months apart. During this interval, some antennas were switched off and the administrators or staff installed new antennas, i.e., noise was introduced. This gave rise to the fingerprint reference database as described in section 5.1.

The SmartUJI Indoor Positioning System (IPS) was evaluated using the 1111 validation samples (from a total of 21048 fingerprints) included in the UJIIndoorLoc database and two different positioning systems: the simplest IPS based on 1-Nearest Neighbor in combination with the Euclidean Distance, and the novel SmartUJI algorithm. The results are summarized in Table 1, and include Success Rate, Average Error, Time and Type of comparison.

Table 1: Results of the Indoor Positioning System (IPS). Simplest IPS (first implemented version) vs. SmartUJI current IPS.

\begin{tabular}{ccc}
\hline & Simplest IPS (INN) & SmartUJI current IPS \\
\hline Average Error (m.) & 7.9 & 3.94 \\
Success Rate (\%) & $89.92 \%$ & $95.05 \%$ \\
Time* (ms.) & $495.29 \pm 0.54$ & $99.12 \pm 0.10$ \\
Type of comparison & Full database & Sector records \\
\hline
\end{tabular}

"Time was calculated after repeating the experiments 20 times. The experiments were run in Matlab on an Intel's Core i7 based computer.

The Success Rate corresponds to the percentage of validation fingerprints correctly located inside the corresponding campus sector. The Average Error is the average error in meters of the validation fingerprints correctly located inside their corresponding campus sector. The Time field contains the average time in milliseconds required to obtain the precise location (x, y, and floor) per fingerprint. The Type of comparison denotes how the fingerprints are compared with the database of reference points: Full database means that a fingerprint is compared to all the reference fingerprints included 
in the database, whereas Sector records denotes the case where a fingerprint is compared only to the fingerprints that were taken in the campus sector provided by the probabilistic procedure.

According to the results shown in Table 1, the proposed location algorithm provides better results in positioning in terms of error and success. Not only has the positioning error been reduced by half ( 4 meters), but the success rate has been considerably improved (5\% increase; total 95\%). Given the challenging scenario described in the beginning of this section, the positioning error can be considered low, and is sufficient to support context-aware mobile applications, such as the ones included in the SmartUJI suite. Moreover, take note that the computational costs of the traditional 1-NN approach were considerably higher. The SmartUJI approach is sector-focused so the computational costs do not depend on the campus' size and only depend on the number of fingerprints inside a sector, that is, 4000 on average. In other words, our IPS has been shown to be scalable.

\subsection{IPS: Discussion}

After analyzing the results, we noticed that the positioning error depended on: 1) the variable nature of the Wi-Fi signal propagation, 2) the Wi-Fi coverage, 3) the mapping strategy, and 4) the hardware and users that collected the fingerprints.

The propagation of Wi-Fi varies through time and space because of the presence of people in corridors, local conditions (open/closed windows and doors), hardware, and orientation, among others. However, considering all the possible cases is nearly impossible and would require taking hundreds or thousands of fingerprints at each reference point. Therefore, the resources required to generate the reference fingerprint database and the operational phase's computational costs were balanced. 
The Wi-Fi coverage is also an important factor influencing accuracy. In fact, there were some isolated research laboratories with low (or null) Wi-Fi coverage; as a result, precise location could not be provided inside them. The presence of nearby wireless access points is a requirement for positioning.

The error in location increased in those places where the mapping procedure was not run or the data density was not high enough. For instance, no training fingerprint were taken inside the restaurant as it was closed for maintenance during the mapping procedure; however it was open when the validation fingerprints were captured. As a result, the error of the validation captures done inside the restaurant is high, as the IPS provides a position outside the restaurant in lack of reference fingerprints inside.

Finally, the current evaluation was realistic since the validation fingerprints were taken four months after the training ones. Moreover, new devices and users participated to collect the validation fingerprints. Using the same device collecting training and testing fingerprints at the same time would have been easier and would have resulted in better accuracy, yet this would not correspond with a real-life use case. Such diversity was deliberately introduced in the UJIIndoorLoc database to simulate a real environment where users, devices and environmental conditions vary.

\subsection{Smart Applications: User experiences and discussion}

Our solutions were deployed in a real-life environment, namely the Universitat Jaume I campus (see section 3), due to its similarity to a small city. To get a first idea of the performance of our mobile applications for mobility, we provided the two applications, SmartUJI APP and SmartUJI AR, to attendees of the 17th AGILE conference on Geographic Information Science attendees (150 people approx.). This conference was hosted by our research group and it took place on the UJI campus. The SmartUJI APP was extended to include a chat room, an activities and sessions calendar, and other 
useful information for the conference (e.g., hotel and gala dinner location).

The conference attendees used and tested both applications, without any restriction or limitation, to retrieve information about the venue facilities and for wayfinding. After the conference, user feedback was collected, which showed that both applications helped users to find the rooms and other points of interest where the conference events took place, and generally their experience was positive. In particular, SmartUJI APP was useful to plan the sessions to attend in advance, and SmartUJI AR allowed retrieving information about the surrounding rooms and facilities in an immersive interface. Moreover, the attendees reported that both applications helped them in finding their way to specific locations, e.g., reaching the plenary room after registration. Attendees also suggested a few minor improvements.

Next to their use by Agile 2014 participants, the two mobile applications have also been tested by university staff and a heterogeneous group of students. These testers reported, after using both applications, that they were useful for finding a classroom, a lab or a professor's office. In particular, academic staff and students found the SmartUJI $A P P$ interesting for scheduling classes in advance. SmartUJI APP helped them to know the location of the classes and estimate the amount of time it takes to travel from one class to another. In general, the SmartUJI AR was considered a good tool to retrieve information about the nearby facilities and improve orientation in the university campus.

Finally, we would like to note that the presented mobile applications are more than just a way to show information and provide wayfinding. They can also serve as analysis tools for managers in the decision-making processes. For example, teaching managers can schedule courses according to the availability and location of facilities. Informal feedback collected from university managers confirm that the SmartUJI 
applications indeed have improved the campus management and optimized the use of resources. Thus, the use case at our university can be considered a successful deployment of a smart platform and applications in a small-medium size environment.

\section{Conclusions}

In this research we aimed to improve mobility in a smart campus setting, which serves as a surrogate for a smart city. We focused on a key issue for mobility: user positioning. We presented a novel indoor positioning system that does not require any additional infrastructure, and is based on existing wireless access point fingerprints. The algorithm was deployed as a service in the SmartUJI platform, a smart campus platform that unifies access to university data sources, and provides services to application developers. We introduced two end-user mobile applications utilizing our Indoor Positioning System, along with other SmartUJI services, to improve mobility in a smart campus scenario. The applications assist users in obtaining location and context-aware information about the university, and allow indoor/outdoor pedestrian wayfinding with maps (SmartUJI APP) or augmented reality (SmartUJI AR). Both the indoor positioning system and the two mobile apps were successfully deployed and evaluated in a realworld environment. A realistic evaluation of our IPS, under real-world conditions, shows positive results regarding accuracy and an averaged positioning error lower than 4 meters. Regarding the mobile apps, experience reports, obtained in several evaluation scenarios from users show they were positively received, and confirm their usefulness for improving mobility. In particular, the SmartUJI APP was perceived useful for planning and scheduling in advance, whereas SmartUJI AR allows the users to interact with the surrounding facilities when they are in the university campus.

As future work, we are continuously developing new services required by students, staff and university managers; and we are also optimizing the existing 
services. Moreover, we are creating an application programming interface (API) for developing mobile applications based on SmartUJI platform. Finally, the next step is to extend this platform to fit in medium-large \& large urban areas.

\section{Acknowledgements}

This work was supported by Universitat Jaume I under the project "Healthy Cities" (Ciudades Saludables, Pla Propi Investigació UJI, P1·1B2012-40), Ministerio de Economía y Competitividad under the project "Smart Ways" (Convocatoría RetosColaboración, RTC-2014-1466-4), and European Commission under the project "ENAbLE" (Erasmus,+ KA2, 8VG-SPS-NW-14-000714-3).

We would like to thank all the current and past members of the Geospatial Research Group for their help in creating the SmartUJI platform and supporting services. We also thank Javier Fernandez, Ángel Ramos, Álvaro Arranz and Guillermo Amat for their collaboration and comments, as members of the Project "Percepción" (Ministerio de Industria, Energía y Comercio, Programa Avanza2, TSI-020601-2012$50)$.

\section{References}

Bache, K., and Lichman, M., 2013. UCI machine learning repository. Available from: http://archive.ics.uci.edu/ml [Accessed date May 2015]

Bahl, P., and Padmanabhan, V.N., 2000. Radar: an in-building rf-based user location and tracking system. In Proceedings of the Nineteenth Annual Joint Conference of the IEEE Computer and Communications Societies vol. 2, 775-784.

Borja, J., 2007. Counterpoint: Intelligent cities and innovative cities. Universitat Oberta de Catalunya (UOC) Papers: E-Journal on the Knowledge Society, 5.

Chourabi, H., Nam, T., Walker, S., Gil-Garcia, J.R., Mellouli, S., Nahon, K., Pardo, T., and Scholl H.J., 2012. Understanding Smart Cities: An Integrative Framework. In Proceedings of the 45th Hawaii International Conference on System Sciences, 2289-2297. 
Chung, J., Donahoe, M., Schmandt, C., Kim, I.J., Razavai, P., and Wiseman, M., 2011. Indoor location sensing using geo-magnetism. In Proceedings of the 9th International Conference on Mobile Systems, Applications, and Services, 141154.

Coccoli, M., Guercio, A., Maresca, P., and Stanganelli, L., 2014. Smarter universities: A vision for the fast changing digital era. Journal of Visual Languages \& Computing, 25(6), 1003-1011.

Cohen, B., 2012. The Top 10 Smart Cities on the Planet. Available from: http://www.fastcoexist.com/1679127/the-top-10-smart-cities-on-the-planet [Accessed date May 2015]

Cover, B., and Hart, P., 1967. Nearest neighbor pattern classification. IEEE Trans. Inf. Theory, 13 (1), 21-27

Feldmann, S., Kyamakya, K., Zapater, A., and Lue, Z., 2003. An indoor bluetoothbased positioning system: Concept, implementation and experimental evaluation. In Proceedings of the International Conference on Wireless Networks, 109-113.

Gansemer, S., Großmann, U., and Hakobyan, S., 2010. RSSI-based Euclidean Distance Algorithm for Indoor Positioning Adapted for the Use in Dynamically Changing WLAN Environments and Multi-level Buildings. In proceedings of the 1st the International Conference on Indoor Positioning and Indoor Navigation.

Giffinger, R., Fertner, C., Kramar, H., Kalasek, R., Pichler-Milanovic, N., and Meijers, E., 2007. Smart Cities - Ranking of European medium-sized cities. Vienna University of Technology. Available from: http://www.smart-cities.eu/download/smart_cities_final_report.pdf [Accessed date May 2015]

Giffinger, R., Kramar, H., and Haindl, G., 2008. The role of rankings in growing city competition. In Proceedings of the 11th European Urban Research Association.

Granell, E., Martínez-Hinarejos, C.D., Amat, G., Fernández, J., Arranz, A., Ramos, A., Benedí, J.M., and Sanchis, A., 2014. Speech Recognition on the Percepción Project. In Proceedings of IberSpeech, 321-330.

Greco, I., and Bencardino, M., 2014. The Paradigm of the Modern City: SMART and SENSEable Cities for Smart, Inclusive and Sustainable Growth. In Proceedings of the 14th International Conference on Computational Science and Its Applications, Lecture Notes in Computer Science vol.8580, 579-597. 
Ijaz, F., Yang, H.K., Ahmad, A.W., and Lee, C., 2013. Indoor positioning: A review of indoor ultrasonic positioning systems. In proceedings of the 15th International Conference on Advanced Communication Technology, 1146-1150.

Jin, G.Y., Lu, X.Y, and Parkm M.S, 2006. An indoor localization mechanism using active RFID tag. In proceedings of the IEEE International Conference on Sensor Networks, Ubiquitous, and Trustworthy Computing.

Kuo, Y. S., Pannuto, P., Hsiao, K. J., and Dutta, P., 2014. Luxapose: Indoor positioning with mobile phones and visible light. In Proceedings of the 20th Annual International Conference on Mobile Computing and Networking, 447-458.

Machaj, J., Brida, P., and PicheÌ, R., 2011. Rank based fingerprinting algorithm for indoor positioning. In Proceedings of the 2nd the International Conference on Indoor Positioning and Indoor Navigation, 1-6.

Marceau, J. (2008). Introduction: Innovation in the city and innovative cities. Innovation: Management, Policy \& Practice, 10(2-3), 136-145.

Marques, N., Meneses, F., and Moreira, A., 2013. Combining similarity functions and majority rules for multi-building, multi-floor, Wi-Fi positioning. In Proceedings of the 3th the International Conference on Indoor Positioning and Indoor Navigation.

Martí, J.V., Sales, J., Marín, R., and Jiménez-Ruiz, E., 2012. Localization of mobile sensors and actuators for intervention in low-visibility conditions: The zigbee fingerprinting approach. International Journal of Distributed Sensor Networks.

McCarthy, F., and Vickers, M., 2008. Digital natives, dropouts and refugees: Educational challenges for innovative cities. Innovation: Management, Policy \& Practice, 10(2-3), 257-268.

Nam, T., and Pardo, T. A., 2011. Smart city as urban innovation: focusing on management, policy, and context. In Proceedings of the 5th International Conference on Theory and Practice of Electronic Governance, 185-194.

Ng, J.W.P., Azarmi, N., Leida, M., Saffre, F., Afzal, A., and Yoo, P.D., 2010. The Intelligent Campus (iCampus): End-to-End Learning Lifecycle of a Knowledge Ecosystem. In Proceedings of the 6th International Conference on Intelligent Environments, 332-337.

Owoc, M., and Marciniak, K., 2013. Knowledge management as foundation of smart university. In Proceedings of the Federated Conference on Computer Science and Information Systems, 1267-1272. 
Torres-Sospedra, J., Montoliu, R., Martinez-Uso, A., Avariento, J. P., Arnau, T. J., Benedito-Bordonau, M., and Huerta, J., 2014. UJIIndoorLoc: A new multibuilding and multi-floor database for WLAN fingerprint-based indoor localization problems. In Proceedings of the Fifth Conference on Indoor Positioning and Indoor Navigation.

Washburn, D., Sindhu, U., Balaouras, S., Dines, R. A., Hayes, N. M., and Nelson, L. E., 2010. Helping CIOs Understand "Smart City" Initiatives: Defining the Smart City, Its Drivers, and the Role of the CIO. Cambridge, MA: Forrester Research, Inc. 


\section{LIST OF FIGURES}

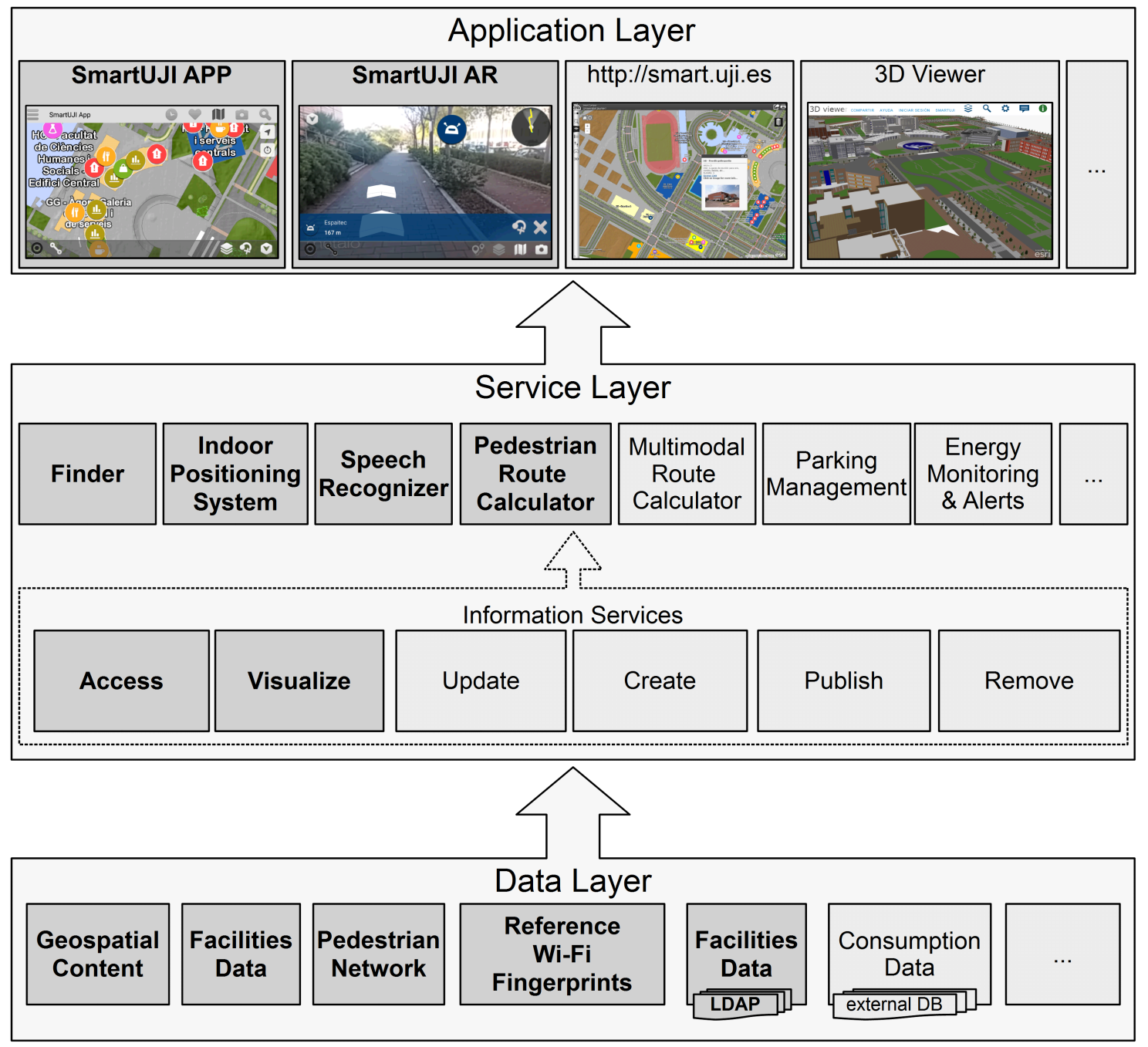

Figure 1: Three layer architecture for SmartUJI. Highlighted in bold are the data sources (internal and external) and services that have been used in the two applications introduced in this paper. 


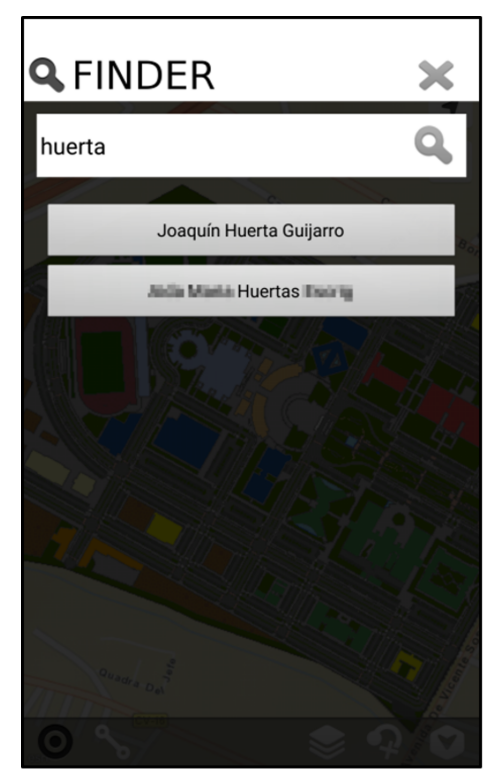

(a)

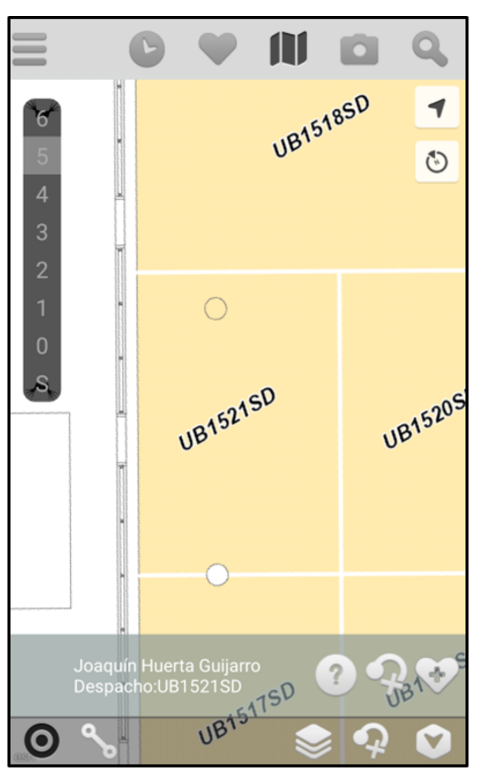

(b)

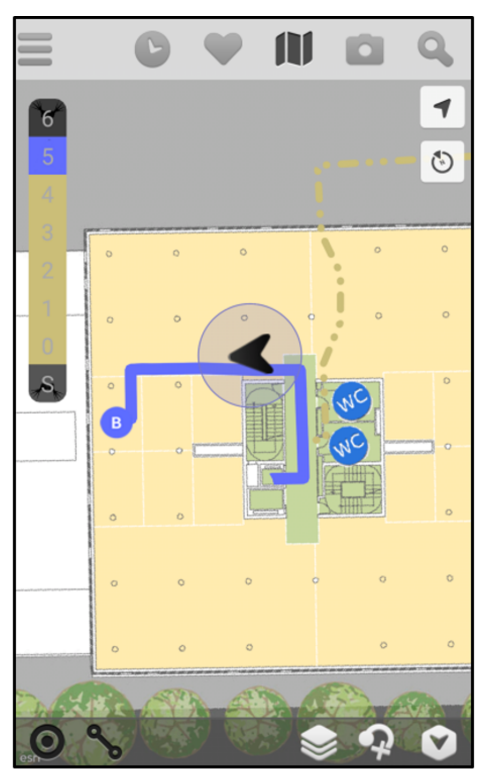

(c)

Figure 2: Screenshots of SmartUJI APP

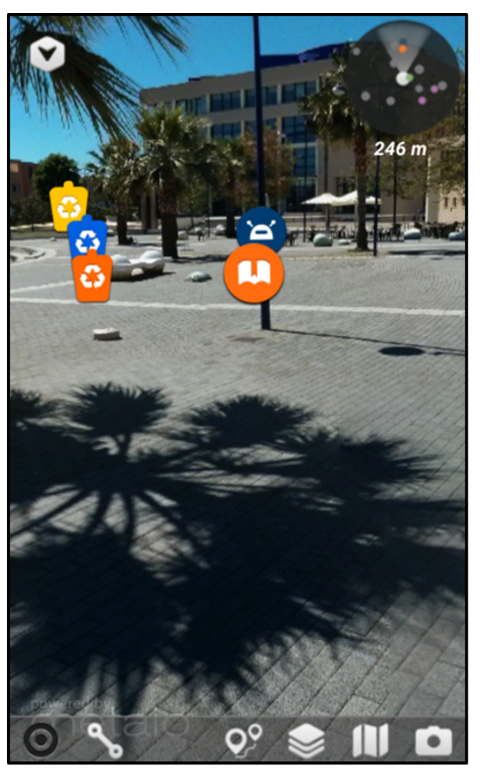

(a)

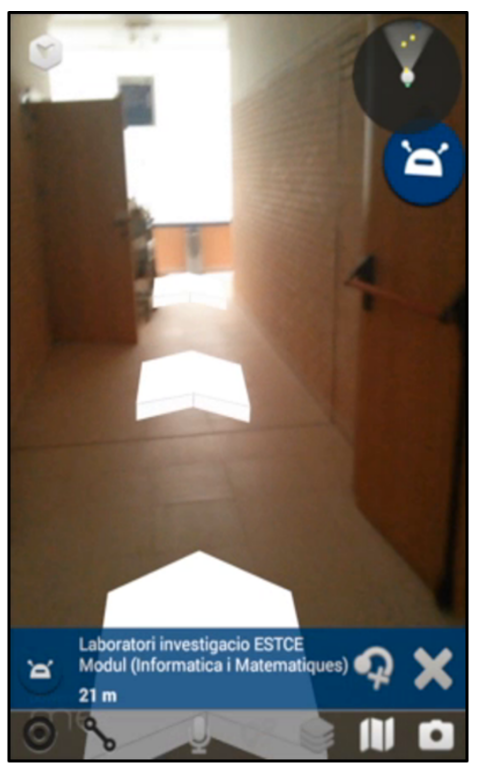

(b)

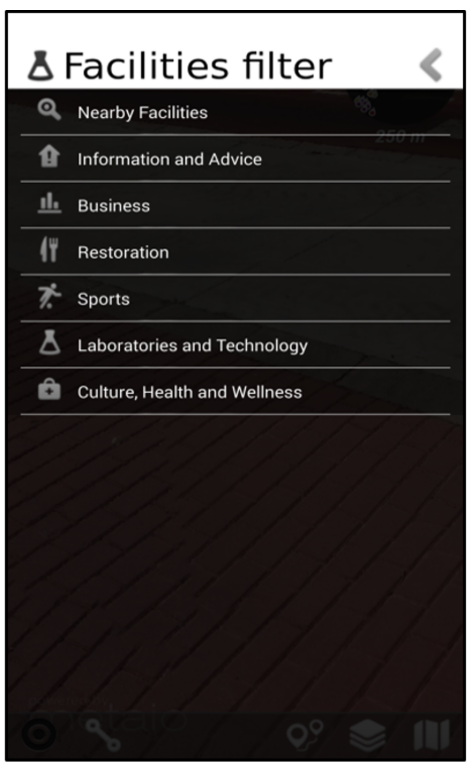

(c)

Figure 3: Screenshots of SmartUJI AR 